\title{
RUNX3 is inactivated by promoter hypermethylation in malignant transformation of ovarian endometriosis
}

\author{
CUISHAN GUO, FANG REN, DANBO WANG, YAN LI, KUIRAN LIU, SHUANG LIU and PENG CHEN \\ Department of Obstetrics and Gynecology, Shengjing Hospital Affiliated to China Medical University, \\ Shenyang, Liaoning 110004, P.R. China
}

Received May 5, 2014; Accepted August 1, 2014

DOI: $10.3892 /$ or.2014.3524

\begin{abstract}
The aim of the present study was to investigate the role of epigenetic inactivation of the runt-related transcription factor 3 gene (RUNX3) in the malignant transformation of ovarian endometriosis. Samples obtained by microdissection and scraping included 30 malignant ovarian endometriotic cyst tissues and 30 corresponding eutopic endometrium tissues from the endometriosis-associated ovarian carcinoma (EAOC) group, 19 benign ovarian endometriotic cyst tissues and 22 corresponding eutopic endometrium tissues from the endometriosis (EM) group and 22 normal eutopic endometrium tissues from the control endometrium (CE) group. RUNX3 methylation status was determined by methylationspecific PCR and bisulfite sequencing, while levels of RUNX3 and ER $\alpha$ protein expression were evaluated using immunohistochemistry. The percentage of RUNX3 methylation and negative RUNX3 protein expression in the malignant ovarian endometriotic cysts from the EAOC group was significantly higher than that in the benign ovarian endometriotic cysts from the EM group. The percentage of RUNX3 methylation and negative RUNX3 protein expression in the eutopic endometrium from the EAOC group was significantly higher than that in the EM and CE groups. An inverse correlation between positive RUNX3 protein expression and methylation was observed and a positive correlation was shown between RUNX3 methylation and $\mathrm{ER} \alpha$ protein expression. In the malignant ovarian endometriotic cysts from the EAOC group, there was no significant correlation between methylation frequency of the RUNX3 gene and histological type. However, the percentage of RUNX3 gene methylation was significantly higher in the tissue samples from patients with surgical stage
\end{abstract}

Correspondence to: Professor Danbo Wang, Department of Obstetrics and Gynecology, Shengjing Hospital Affiliated to China Medical University, 36 Sanhao Street, Shenyang, Liaoning 110004, P.R. China

E-mail:wangdb@sj-hospital.org

Key words: RUNX3, endometriosis, malignant transformation of ovarian endometriosis, ovarian carcinoma, hypermethylation, eutopic endometrium
IC EAOC than the percentage in patients with stage IA and IB disease. These results suggest that RUNX3 inactivation by promoter hypermethylation plays a role in the progression of malignant transformation of ovarian EM and is closely related to estrogen metabolism. Negative protein expression and abnormal RUNX3 methylation in the eutopic endometrium could be used as diagnostic markers in patients with ovarian EM who may be at an increased risk of developing EAOC.

\section{Introduction}

Endometriosis is an estrogen-dependent gynecological disorder that has many causes and is associated with many genes. It is estimated that endometriosis may affect between 5 and $10 \%$ of women who are of reproductive age (1). While endometriosis is a benign lesion, it shares several characteristics with invasive cancer. Similar to cancer, its behavior is characterized by proliferation, invasion, metastasis and a high recurrence rate. Since Sampson first theorized that endometriotic lesions could undergo malignant transformation in 1925 (2), histological and epidemiological studies have suggested that ovarian endometriosis can transform into ovarian carcinoma. The average rate of endometriotic malignant transformation is $\sim 0.7 \%$ (3). With an increase in the morbidity associated with endometriosis, the malignant transformation rate also has increased. Stern et al (4) showed a 5\% incidence of ovarian malignancy associated with ovarian endometriosis. According to Yoshikawa et al (5), types of malignancies in ovarian endometriosis include clear cell (39.2\%), endometrioid (21.2\%), serous (3.3\%) and mucinous $(3.0 \%)$. Currently, endometrioid and clear cell carcinomas are the predominant histological subtypes of endometriosis-associated ovarian carcinoma (EAOC). Ovarian cancer is a malignant disease with high global morbidity and mortality rates. Research on the pathogenesis and candidate genes involved in EAOC development is important for early detection, prevention and identification of new therapeutic targets.

Research regarding the mechanisms involved in EAOC has been limited by the low number of cases, difficulty obtaining pathological materials and other factors. In recent years, information concerning the pathogenesis of ovarian cancer has significantly changed, including introduction of a dualistic model in which the majority of what appear to be primary ovarian cancers, namely serous, endometrioid and clear cell 
carcinomas, are thought to be derived from the fallopian tube and endometrium rather than directly from the ovary (6). Understanding the associations between endometrioid and clear cell carcinomas and malignant transformation of ovarian endometriosis will require new approaches. At present, the rate of endometriotic malignant transformation may be underestimated, and evidence-based medicine and epidemiology are necessary for revalidation. Fundamental research regarding malignant transformation of endometriosis will also require further attention.

Tumor-suppressor genes can be inactivated through a variety of mechanisms. In addition to genetic mutations or deletions, DNA methylation is widely considered one of the mechanisms that leads to decreased genetic expression. Research has shown that DNA methylation may represent an early, fundamental step in the pathway through which normal tissues undergo neoplastic transformation (7). Assessment of methylation profiles within neoplastic tissues may provide key information in enhancing diagnosis, predicting clinical behavior and designing specific treatment plans for individual patients. Martini et al (8) demonstrated that hMLH1 promoter hypermethylation and resultant gene silencing may be involved in the malignant transformation of endometriosis. We previously screened candidate genes related to malignant transformation of ovarian endometriosis for aberrant methylation by evaluating $\mathrm{CpG}$ island methylation using enrichment methods combined with representational difference analysis (MCA-RDA). RUNX3 was one of the 9 candidate genes and is a tumor-suppressor gene located on 1p36 (9). The largest, most recent genetic studies of endometriosis and EAOC have identified $1 \mathrm{p} 36$ as a region of paramount significance (10). The runt domain family, consisting of RUNX1, RUNX2 and RUNX3, are master regulators of gene expression in cell proliferation and differentiation. All three RUNX family members play important roles in normal developmental processes and carcinogenesis (11). Among the three RUNX family members, RUNX3 was originally cloned as AML2 and was first reported to be correlated with the genesis and progression of human gastric cancer as a tumor suppressor (12). The RUNX3 protein can mediate the growth suppressive effects of TGF- $\beta$ by associating with SMAD, a downstream protein in the signaling pathway (13). RUNX3 has been shown to be a target for epigenetic gene silencing via aberrant hypermethylation of its $\mathrm{CpG}$ island promoter region in a variety of estrogendependent tumors, such as breast cancer (14), endometrial cancer (15), ovarian granulosa cell tumors (GCTs) (16) and testicular yolk sac tumors in infants (17). Cheng et al (18) found that RUNX3 promoter hypermethylation was induced by estrogen in mammosphere-derived breast cancer cells. This estrogen-dependent epigenetic silencing of RUNX3 is likely mediated by estrogen receptor $\alpha(E R \alpha)$ signaling. Estrogen, a major trigger for malignant transformation of endometriosis, appears to play a role in this process. Prolonged exposure to estrogen is known to increase the risk of developing malignant transformation of endometriosis (19). We hypothesized that RUNX3 inactivation by promoter hypermethylation is involved in malignant transformation of endometriosis, which has not been previously reported.

In the present study, we investigated the association between RUNX3 inactivation primarily due to promoter methylation and malignant transformation of ovarian EM. We also investigated whether RUNX3 detection in the eutopic endometrium could be used as a diagnostic marker in patients with endometriosis who may be at an increased risk of developing EAOC. In addition, we evaluated the relationship between RUNX3 promoter methylation and ER $\alpha$ protein expression to define the role of estrogen in initiating RUNX3 promoter methylation.

\section{Materials and methods}

Patients and materials. A total of 74 formalin-fixed and paraffin-embedded (FFPE) samples were obtained from the Department of Pathology at Shengjing Hospital, China Medical University from January 2005 to December 2013. Malignant ovarian endometriotic cysts and the corresponding eutopic endometrium from 30 patients who had undergone total hysterectomy or salpingo-oophorectomy and surgical staging of ovarian cancer (13 endometrioid and 17 clear cell cancers) due to EAOC were collected as the EAOC group. Histopathological examination revealed the coexistence and continuity of endometriosis and cancer in patients with EAOC, fulfilling the criteria proposed by Sampson (2) and Scott (20) (Fig. 1). Benign ovarian endometriotic cysts and the corresponding eutopic endometrium from 22 patients who had undergone total hysterectomy due to ovarian EM were collected as the EM group. The normal eutopic endometrium from 22 patients diagnosed with benign uterine conditions without endometriosis and estrogen-dependent diseases who had undergone total hysterectomy were collected as the $\mathrm{CE}$ group. The average patient age in the EAOC, EM and CE group was $51.17 \pm 1.50,47.68 \pm 0.64$ and $47.36 \pm 0.40$ years, respectively, and there was no statistical difference in age among the three groups $(\mathrm{P}>0.05)$. None of the patients had received gonadotropin releasing hormone $(\mathrm{GnRH})$ analogs or other hormonal medications or antibiotics during the 6 months prior to surgery. Histological diagnoses for each patient were reviewed by a gynecological pathologist following the criteria of the World Health Organization classification.

Microdissection. All paraffin-embedded tissues were sliced at a 5- $\mu \mathrm{m}$ thickness for hematoxylin and eosin (H\&E) staining and consecutively sliced into 4-6 sections at a 10- $\mu \mathrm{m}$ thickness unstained for microscopic cutting. Microscopic cutting steps were as follows. One slide of each specimen was randomly selected for H\&E staining as a template; the other slides were placed on the H\&E template and the target area was scraped using a disposable sterile blade under a dissection microscope. Scraped tissues included 30 malignant ovarian endometriotic cysts and 30 corresponding eutopic endometrium tissues from the EAOC group, 19 benign ovarian endometriotic cysts and 22 corresponding eutopic endometrium tissues from the EM group and 22 normal eutopic endometrium tissues from the $\mathrm{CE}$ group. Of the 22 patients who were histologically confirmed as having ovarian EM, 3 patient samples did not have sufficient tissue for microscopic cutting.

DNA extraction and bisulfite modification. Total genomic DNA was extracted from $10-\mu \mathrm{m}$ paraffin tissue sections using the TIANamp Micro DNA kit (Tiangen Biotech Co., Ltd., 


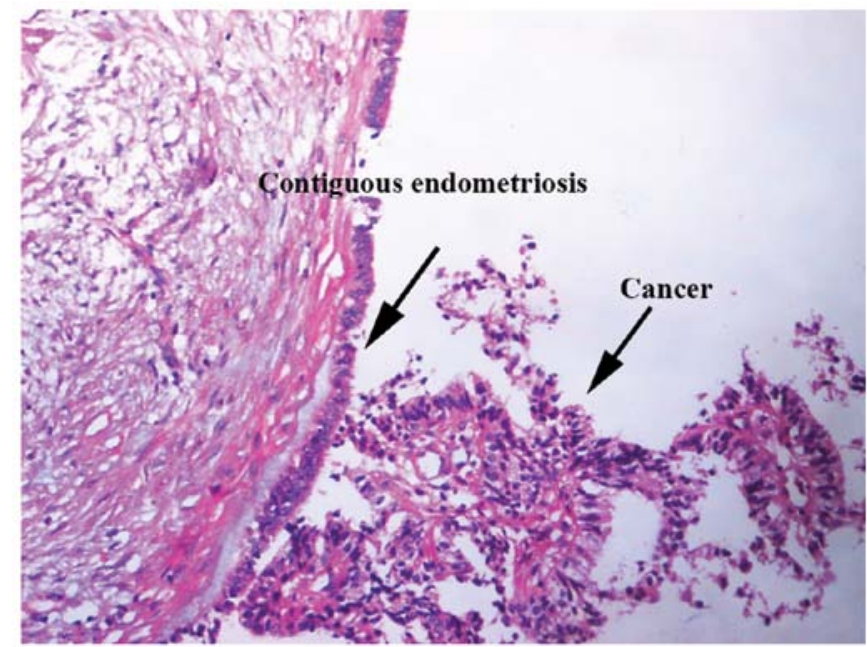

Figure 1. Histologic image of EAOC (H\&E staining). The image shows the transitional area from ectopic endometrium to cancer and the ovarian cancer area (original magnification, x200). EAOC, endometriosis-associated ovarian carcinoma; H\&E staining, hematoxylin and eosin staining.

Beijing, China) according to the manufacturer's instructions, and $0.5 \mu \mathrm{g}$ from each sample was modified via sodium bisulfite treatment using the EZ DNA Methylation-Gold kit (Zymo Research Corp., Orange, CA, USA) following the manufacturer's protocol.

Methylation-specific PCR (MSP) and sequencing (BSP). Methylation status in the RUNX3 promoter region was determined by MSP. Modified genomic DNA was amplified using two different sets of primers specific for unmethylated and methylated RUNX3 promoters. Primers for the methylated reaction were as follows (3): sense, 5'-ATA ATA GCG GTC GTT AGG GCG TCG-3' and antisense, 5'-GCT TCT ACT TTC CCG CTT CTC GCG-3'. Primers for the unmethylated reaction were as follows: sense, 5'-ATA ATA GTG GTT GTT AGG GTG TTG-3' and antisense, 5'-ACT TCT ACT TTC CCA CTT CTC ACA-3' and the product size was $115 \mathrm{bp}$. PCR was performed under the following conditions: initial denaturation at $94^{\circ} \mathrm{C}$ for 5 min followed by 35 amplification cycles (denaturation at $94^{\circ} \mathrm{C}$ for $30 \mathrm{sec}$, annealing at $55^{\circ} \mathrm{C}$ for $30 \mathrm{sec}$ and extension at $72^{\circ} \mathrm{C}$ for $30 \mathrm{sec}$ ) and a final extension at $72^{\circ} \mathrm{C}$ for $7 \mathrm{~min}$ in a PCR Thermal Cycler Dice (Takara Bio Inc., Otsu, Japan) using the Takara EpiTap ${ }^{\mathrm{TM}}$ HS kit (Takara Biotechnology Co., Ltd., Dalian, China). Normal lymphocyte DNA methylated in vitro with SssI methylase (New England Biolabs, Beverly, MA, USA) was used as a positive control $(\mathrm{M}+)$ and water was used as a blank control. Products were subjected to electrophoresis on a $2 \%$ agarose gel. For sequencing, a 414-nt region of the RUNX3 promoter between -797 and -384 relative to the translation start site (TSS) was amplified with the Takara EpiTap ${ }^{\mathrm{TM}}$ HS kit using primers: forward, AGAAGTAGTAAGAGTTGGGGAAGTT and reverse, ACAAATCCTCCAAAATCAAATAAC. Amplicons were gel purified using the TIANgel Midi Purification kit (Tiangen Biotech Co., Ltd.) and subcloned into the PCR $^{\circledR}$ 2.1 vector (Invitrogen). Five clones were randomly selected, sequenced and analyzed using a BISMA analyzer.

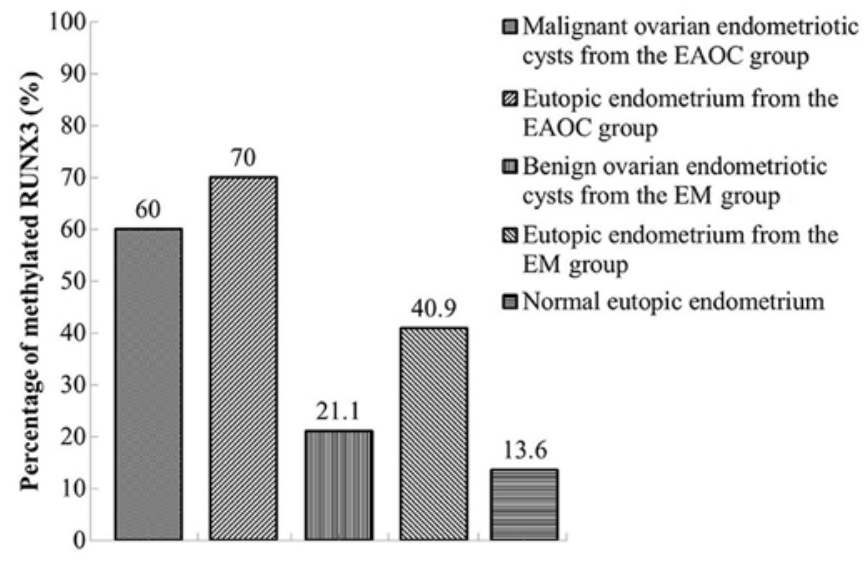

Figure 2. Comparison of RUNX3 methylation frequencies among the EAOC, EM and CE groups. RUNX3, runt-related transcription factor 3 gene; EAOC, endometriosis-associated ovarian carcinoma; EM, endometriosis; CE, control endometrium.

Immunohistochemistry. All FFPE tissues were sectioned at a thickness of $5-\mu \mathrm{m}$ for immunohistochemical staining using the standard streptavidin-biotin method. Slides were dewaxed and rehydrated following standard procedures. Antigen retrieval was performed to enhance immunohistochemistry by microwaving the slides in citrate buffer $(\mathrm{pH} \mathrm{6.0)}$ for $10 \mathrm{~min}$. Endogenous peroxidase activity was blocked for $10 \mathrm{~min}$ in $0.3 \% \mathrm{H}_{2} \mathrm{O}_{2}$. Sections were subsequently washed and blocked with normal goat serum for $30 \mathrm{~min}$ at room temperature and incubated with mouse monoclonal antibody to RUNX3 (1:400; Enzo Life Sciences AG, Lausen, Switzerland) and rabbit polyclonal antibody to ER $\alpha$ (1:100; Abcam, Cambridge, UK) overnight at $4^{\circ} \mathrm{C}$. Slides were washed in phosphate-buffered saline and incubated with biotinylated goat anti-mouse or goat anti-rabbit secondary antibodies for $30 \mathrm{~min}$ at room temperature. Antigen-antibody complexes were detected with avidin-biotin peroxidase for $30 \mathrm{~min}$ at room temperature. Slides were stained with diaminobenzidine until the desired staining intensity was developed and observed by light microscopy. Antibody effectors of RUNX3 and ER $\alpha$ are brown granules expressed in the nucleus and/or cell cytoplasm. RUNX3 and ER $\alpha$ expression was evaluated by combining the estimated percentage of positive cells with the estimated staining intensity. The frequency of positively stained cells per 100 cells was scored as 0 (no positive cells), 1 (positive cells $\leq 10 \%$ ), 2 (positive cells $>10 \%$ and $\leq 50 \%$ ), 3 (positive cells $>50 \%$ and $\leq 75 \%$ ) or 4 (positive $>75 \%$ ). Staining intensity was scored as follows: 0 , negative (no color); 1 , weak brown; 2 , moderate brown and 3, strong brown. Raw data were converted to an immunohistochemical score by multiplying the quantity and staining intensity scores and graded from - to +++ (-, $0-4$; $+, 4-5 ;++, 6-8 ;+++, 9-12)$. IHC scoring was performed independently by two pathologists, and their consensus was considered the final score in each case. The immunoreactivity results - and + were considered negative, while ++ and +++ results were considered positive.

Statistical analysis. Statistical analysis was performed with SPSS software version 13.0 (SPSS Inc., Chicago, IL, USA). Results were analyzed using the Chi-squared test and Fisher's 


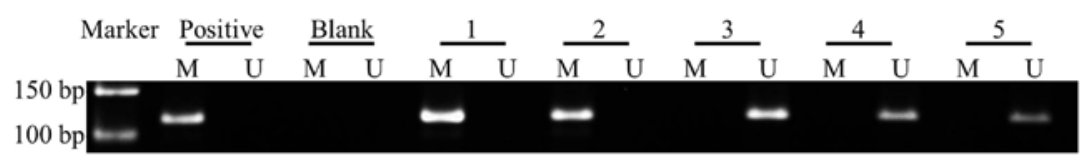

Figure 3. Representative image from MSP analysis of the RUNX3 methylation status in the EAOC group, EM group and CE group. M and U are the methylated and unmethylated primers, respectively. Positive, methylation-positive control; Blank, blank control. Lane 1, malignant ovarian endometriotic cyst tissues from the EAOC group; lane 2, corresponding eutopic endometrium tissues from the EAOC group; lane 3, benign ovarian endometriotic cyst tissues from the EM group; lane 4, corresponding eutopic endometrium tissues from the EM group; lane 5, normal eutopic endometrium from the CE group. RUNX3, runt-related transcription factor 3 gene; EAOC, endometriosis-associated ovarian carcinoma; EM, endometriosis; CE, control endometrium.

Table I. Relationship between RUNX3 methylation in malignant ovarian endometriotic cysts from the EAOC group and histological type and stage.

\begin{tabular}{|c|c|c|c|c|c|}
\hline Characteristics & No. of cases & Methylated & Unmethylated & $\begin{array}{c}\text { Methylated } \\
\text { RUNX3 (\%) }\end{array}$ & P-value \\
\hline \multicolumn{6}{|l|}{ Histological types } \\
\hline Endometrioid carcinoma & 13 & 7 & 6 & 53.8 & 0.7106 \\
\hline Clear cell carcinoma & 17 & 11 & 6 & 64.7 & \\
\hline \multicolumn{6}{|l|}{ Stage } \\
\hline IA & 12 & 5 & 7 & 41.7 & $0.6000^{\mathrm{a}}$ \\
\hline IB & 5 & 1 & 4 & 20.0 & $0.0068^{\mathrm{b}}$ \\
\hline IC & 13 & 12 & 1 & 92.3 & $0.0077^{\mathrm{c}}$ \\
\hline \multicolumn{6}{|l|}{$\mathrm{ER} \alpha$} \\
\hline Positive & 12 & 11 & 1 & 91.7 & 0.0068 \\
\hline Negative & 18 & 7 & 11 & 38.9 & \\
\hline
\end{tabular}

RUNX3, runt-related transcription factor 3 gene; EAOC, endometriosis-associated ovarian carcinoma; ER $\alpha$, estrogen receptor $\alpha$; ${ }^{a} F i s h e r ' s$ exact test, IA vs. IB stage; 'Fisher's exact test, IA vs. IC stage; 'Fisher's exact test, IB vs. IC stage.

exact probability test when appropriate. A two-sided value of $\mathrm{P}<0.05$ was considered statistically significant.

\section{Results}

Increased hypermethylation of the RUNX3 promoter. RUNX3 methylation was detected in 18 of $30(60.0 \%)$ malignant ovarian endometriotic cysts in the EAOC group and 4 of 19 (21.1\%) benign ovarian endometriotic cysts in the EM group. The percentage of RUNX3 gene methylation in the EAOC group was significantly higher than that in the EM group (Fig. 2, $\mathrm{P}<0.05)$. Methylation frequencies in the corresponding eutopic endometrium from the EAOC, EM and $\mathrm{CE}$ groups were $70.0 \%(21 / 30), 40.9 \%(9 / 22)$ and $13.6 \%$ (3/22), respectively. Statistically significant differences in the frequency of RUNX3 gene methylation were observed, with gradually decreasing levels among the three groups (Fig. 2, $\mathrm{P}<0.05$ ). However, this significant difference was not found among the malignant ovarian endometriotic cysts, benign ovarian endometriotic cysts and their corresponding eutopic endometrium ( $\mathrm{P}>0.05)$. Representative images from MSP analysis show methylated and unmethylated PCR products (Fig. 3).

The methylation status of $48 \mathrm{CpGs}$ across a 414-nt region from -797 to -384 relative to the TSS was characterized by bisulfite genomic sequencing, which is the gold standard for mapping methylated $\mathrm{CpG}$ sites. We randomly selected 5 malignant ovarian endometriotic cyst tissues from the EAOC group,
5 benign ovarian endometriotic cyst tissues from the EM group and 5 normal eutopic endometrium from the CE group. After subcloning the PCR products generated from bisulfite-treated DNA using the $\mathrm{PCR}^{\circledR} 2.1$ vector, a selected clone was sequenced to determine the precise methylation status of $48 \mathrm{CpG}$ sites within the RUNX3 gene. Malignant ovarian endometriotic cysts from the EAOC group showed a dense methylation pattern in this region of the RUNX3 gene compared to benign ovarian endometriotic cysts from the EM group. No methylated $\mathrm{CpG}$ sites were detected in 5 samples from the CE group (Fig. 4).

Relationship between RUNX3 methylation in malignant ovarian endometriotic cysts from the EAOC group and histological type and stage. As shown in Table I, there was no significant correlation between the percentage of methylation of the RUNX3 gene and histological type in the malignant ovarian endometriotic cysts from the EAOC group ( $\mathrm{P}>0.05)$. However, the percentage of RUNX3 gene methylation was significantly higher in the tissue samples from patients with surgical stage IC EAOC than this percentage in the patients with stage IA and IB disease $(\mathrm{P}<0.01)$.

Association between RUNX3 methylation status and protein expression. In the EAOC group, the percentage of positive RUNX3 protein expression was markedly higher in the malignant ovarian endometriotic cysts $(36.7 \%)$ than that in the corresponding eutopic endometrium $(10.0 \%)(\mathrm{P}<0.05)$. In the 
A
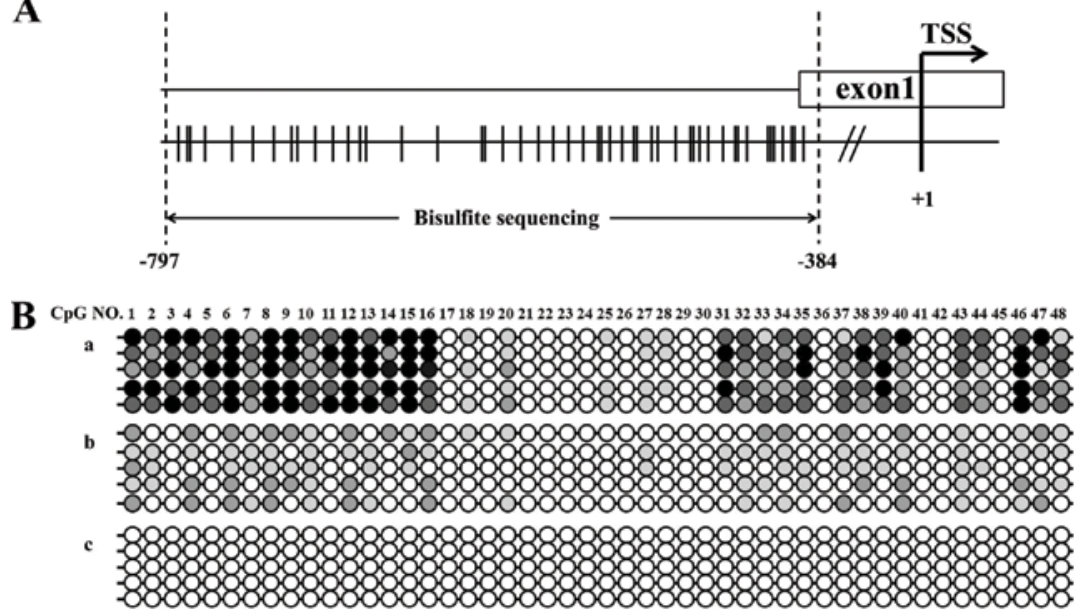

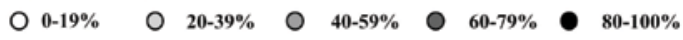

C

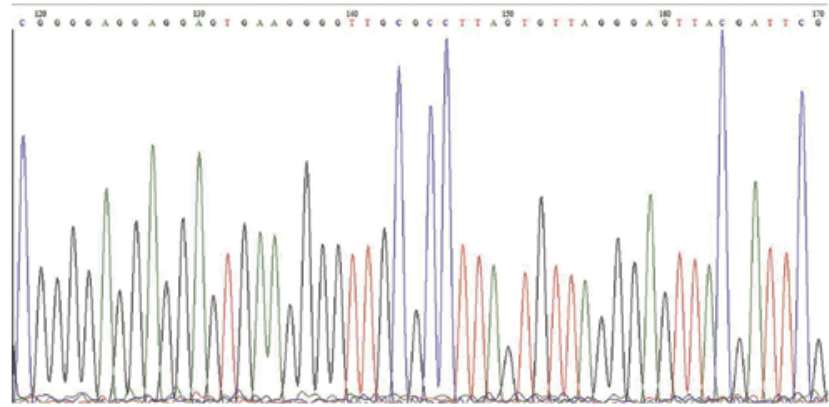

Figure 4. DNA methylation analysis of RUNX3 by BSP. (A) The cloned bisulfite sequencing was performed on the RUNX3 genomic region (-797 to -384) relative to the transcription start site (TSS). A vertical bar indicates a CpG site. (B) The percentage of methylation on each CpG dinucleotide was calculated by the number of methylated clones in each $\mathrm{CpG}$ site divided by the total number of clones in the same $\mathrm{CpG}$ site. A circle indicates a CpG site: a, malignant ovarian endometriotic cyst tissues from the EAOC group; b, benign ovarian endometriotic cyst tissues from the EM group; c, normal eutopic endometrium from the CE group. (C) Representative images demonstrating CpG island methylation status of RUNX3 in malignant ovarian endometriotic cyst tissues from the EAOC group. RUNX3, runt-related transcription factor 3 gene; EAOC, endometriosis-associated ovarian carcinoma; EM, endometriosis; CE, control endometrium.

Table II. Association between RUNX3 methylation status and protein expression.

\begin{tabular}{|c|c|c|c|c|c|c|}
\hline & \multirow[b]{2}{*}{$\begin{array}{l}\text { No. of } \\
\text { cases }\end{array}$} & \multicolumn{3}{|c|}{ RUNX3 methylated } & \multicolumn{2}{|c|}{ RUNX3 unmethylated } \\
\hline & & Total & $\begin{array}{l}\text { Positi } \\
\text { protein } \mathrm{C}\end{array}$ & $\begin{array}{l}\text { ve RUNX3 } \\
\text { xpression (\%) }\end{array}$ & Total & $\begin{array}{c}\text { Positive RUNX3 } \\
\text { protein expression (\%) }\end{array}$ \\
\hline \multicolumn{7}{|l|}{ EAOC group } \\
\hline Malignant ovarian endometriotic cysts & 30 & 18 & 0 & $(0.0)$ & 12 & $11(91.7)$ \\
\hline Eutopic endometrium & 30 & 21 & 1 & $(4.8)$ & 9 & $2(22.2)$ \\
\hline \multicolumn{7}{|l|}{ EM group } \\
\hline Benign ovarian endometriotic cysts & 19 & 4 & & $(25.0)$ & 15 & $15(100.0)$ \\
\hline Eutopic endometrium & 22 & 9 & 3 & $(33.3)$ & 13 & $10(76.9)$ \\
\hline \multicolumn{7}{|l|}{ CE group } \\
\hline Normal eutopic endometrium & 22 & 3 & & $(100.0)$ & 19 & $19(100.0)$ \\
\hline
\end{tabular}

RUNX3, runt-related transcription factor 3 gene; EAOC, endometriosis-associated ovarian carcinoma; EM, endometriosis; CE, control endometrium.

EM group, the percentage of positive RUNX3 protein expression was markedly higher in the benign ovarian endometriotic cysts $(84.2 \%)$ than that in the corresponding eutopic endometrium $(59.1 \%)(\mathrm{P}<0.05)$. Compared to the benign ovarian endometriotic cysts in the EM group (84.2\%), the percentage of positive RUNX3 protein expression in the malignant ovarian endometriotic cysts from the EAOC group (36.7\%) was significantly downregulated $(\mathrm{P}<0.05)$. In the corresponding eutopic 

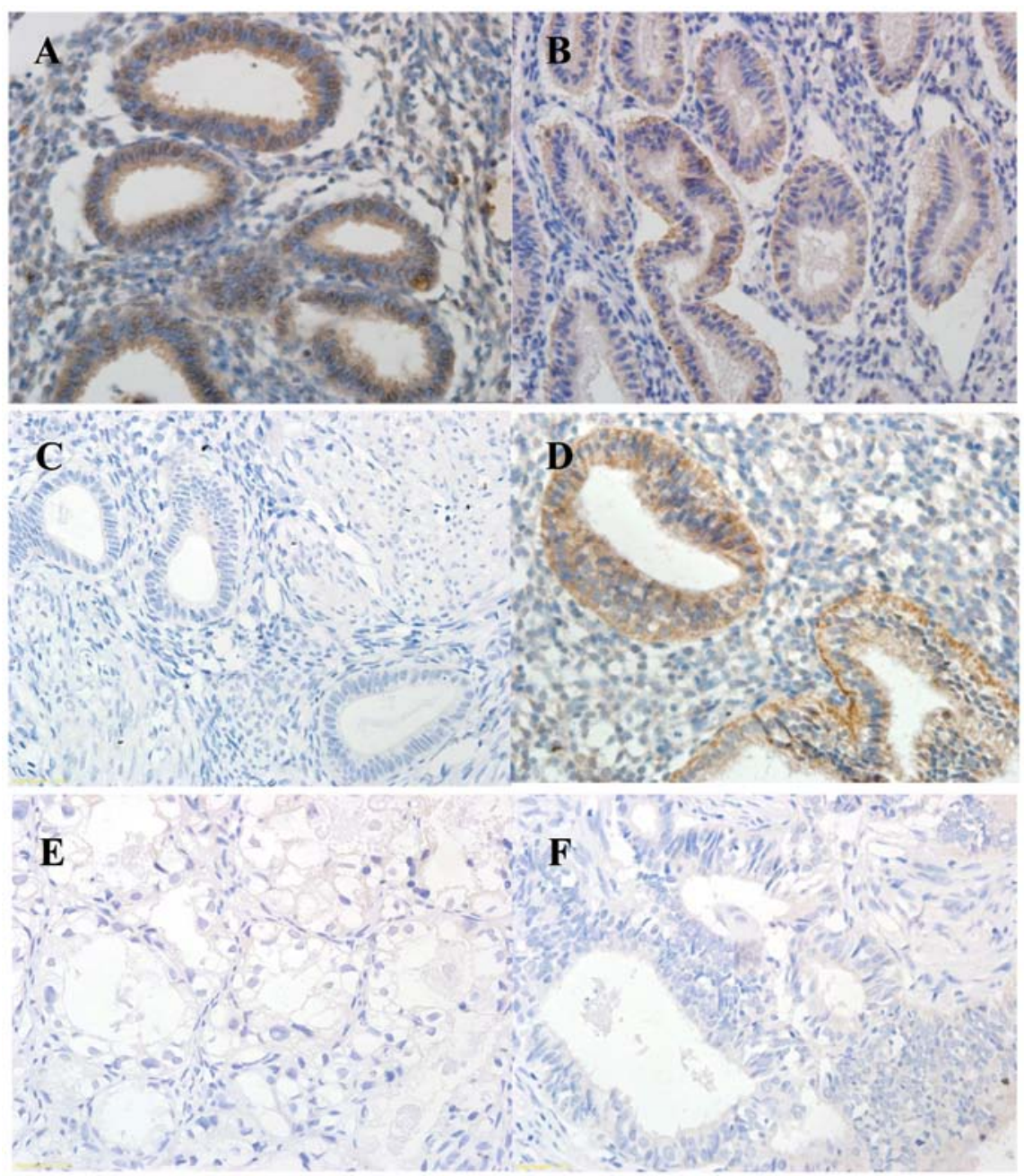

Figure 5. Immunohistochemical evaluation of RUNX3 expression in the EAOC, EM and CE groups. (A) Normal eutopic endometrium from the CE group, (B) corresponding eutopic endometrium tissues from the EM group, (C) corresponding eutopic endometrium tissues from the EAOC group, (D) benign ovarian endometriotic cyst tissues from the EM group, (E) clear cell carcinoma and (F) endometrioid carcinoma (original magnification, $x 400$ ). RUNX3, runt-related transcription factor 3 gene; EAOC, endometriosis-associated ovarian carcinoma; EM, endometriosis; CE, control endometrium.

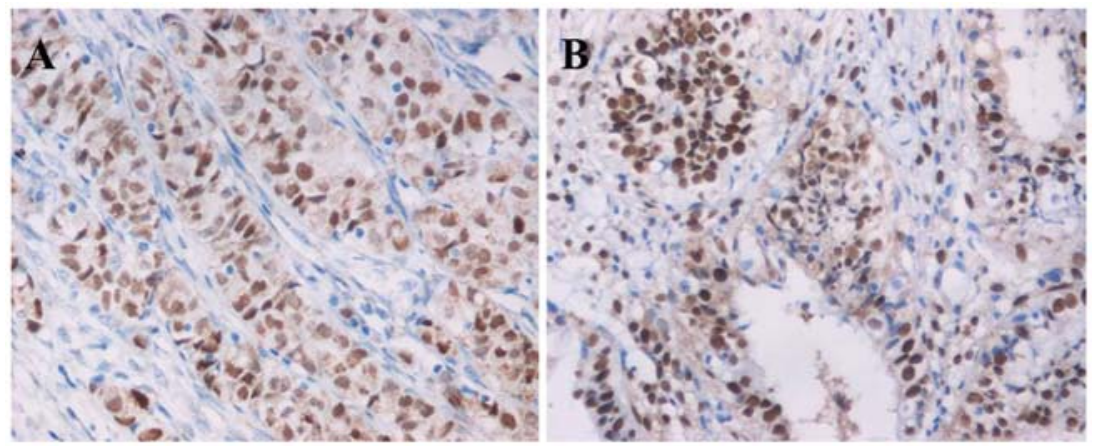

Figure 6. Immunohistochemical staining for ER $\alpha$ in malignant ovarian endometriotic cyst tissues from the EAOC group. (A) Clear cell carcinoma, (B) endometrioid carcinoma (original magnification, $\mathrm{x} 400$ ). $\mathrm{ER} \alpha$, estrogen receptor $\alpha$.

endometrium from the EAOC, EM and $\mathrm{CE}$ groups, the incidence of positive RUNX3 protein expression was 10.0, 59.1 and $100.0 \%$, respectively $(\mathrm{P}<0.05)$ (Fig. 5, Table II).

Overall, 47 of the 55 methylated samples were negative for RUNX3 protein expression and only 11 of the 68 RUNX3 unmethylated samples were negative for RUNX3 protein expression. An inverse correlation was noted between positive RUNX3 protein expression and methylation $\left(r_{s}=-0.69\right.$, $\mathrm{P}<0.01)$ (Table II).

Correlation between RUNX3 methylation status and ERa protein expression in the malignant ovarian endometriotic cysts from the EAOC group. In the malignant ovarian endometriotic cysts from the EAOC group, the methylation 
frequency of the RUNX3 gene was higher in the group with positive ER $\alpha$ protein expression compared to the group with negative ER $\alpha$ protein expression $(\mathrm{P}<0.05)$. Eleven of the 18 RUNX3 methylated samples had positive ER $\alpha$ protein expression, while only 1 of 12 RUNX3 unmethylated samples were positive. A positive correlation was shown between RUNX3 methylation and ER $\alpha$ protein expression $\left(r_{s}=0.53\right.$, $\mathrm{P}<0.01$ ) (Fig. 6, Table I).

\section{Discussion}

Malignant transformation of endometriosis is a long, complicated process involving multiple genes and steps. Epigenetic inactivation of cancer-associated genes has been observed frequently in most human cancers and has been shown to be closely associated with DNA hypermethylation. Silencing the promoter region in CpGs of regulated genes, such as RUNX3, via hypermethylation is recognized as one of three mechanisms that contributes to loss of gene function in Knudson's two-hit tumor progression hypothesis (21). More importantly, silencing due to hypermethylation is an epigenomic change in premalignant lesions as part of the multistep carcinogenetic process in some malignancies (7). DNA methylation is a heritable, enzyme-induced modification to the DNA structure without altering the specific sequence of base pairs responsible for encoding the genome and the abnormal methylation patterns can be reversed (22). Consequently, systemic study of gene methylation is extremely important in the early diagnosis and treatment of diseases. Senthong et al (23) demonstrated that LINE-1 hypomethylation occurs early in EAOC development and indicated that epigenomic alterations may precede development of ovarian cancer phenotypes. Therefore, epigenetic modifications, particularly DNA methylation, provide exciting new avenues in the study of the malignant transformation of endometriosis.

Epigenetic inactivation of RUNX3 has been detected in numerous cancers, particularly malignancies that involve estrogen metabolism. Herein, we report the RUNX3 methylation status in ovarian EM and EAOC for the first time. In the present study, the frequency of RUNX3 methylation and negative RUNX3 protein expression in malignant ovarian endometriotic cysts from the EAOC group was significantly higher than that in the benign ovarian endometriotic cysts from the EM group and normal eutopic endometrium of the CE group. RUNX3 expression was inversely correlated with aberrant hypermethylation of $\mathrm{CpG}$ islands in the RUNX3 promoter region. Inactivation of RUNX3 expression primarily due to frequent promoter hypermethylation may play an important role in transformation of the endometrium to endometriosis and EAOC. In addition, a relatively high percentage of RUNX3 methylation was detected in benign ovarian endometriotic cysts from the EM group and normal eutopic endometrium from the CE group, suggesting that epigenomic alterations in precancerous lesions can result in individuals becoming carriers of abnormal methylation patterns. Although the tissue morphology may appear normal, cells have changed at a molecular level, which has been widely observed in other tumors (24). Therefore, RUNX3 methylation is an early event in the pathogenesis of EAOC and may be a target for early EAOC detection.
Within the same tumor, methylation frequencies at different CpG sites are not exactly the same (25). There are examples in which methylation of individual $\mathrm{CpG}$ sites in promoter regions can determine the transcriptional status of a gene by blocking access of certain transcription factors that are sensitive to $\mathrm{CpG}$ methylation at target binding sites. Alternatively, methylation of sites in these regions may also silence genes by helping to recruit chromatin protein complexes that repress transcription (26). In a previous study, we screened different fragments of RUNX3 methylation using MCA-RDA at sites located from -810 to -582 relative to the TSS. In the present study, we tested the methylation status at a total of $48 \mathrm{CpG}$ sites across a 414-bp region from -797 to -384 relative to RUNX3 TSS. Malignant ovarian endometriotic cysts from the EAOC group showed a dense methylation pattern in this region compared to benign ovarian endometriotic cysts from the EM group. No methylation $\mathrm{CpG}$ sites were detected in 5 samples from the CE group. These results further confirm that methylation of sites in this region play a significant role in the malignant transformation of ovarian EM. Hypermethylation of promoter $\mathrm{CpG}$ islands in tumor suppressors is known to be a frequent, early reversible event in carcinogenesis and can contribute to disease progression (27). Although the frequency of RUNX3 methylation increased with pathological stage, there was no significant correlation with histological type, demonstrating that methylation may play a role in both the occurrence and development of EAOC regardless of histology. These data strongly imply early epigenetic gene inactivation in EAOC and may assist in identifying novel epigenetic biomarkers associated with EAOC risk.

Studies have shown that as many as $50 \%$ of women with ovarian endometrioid carcinomas also have a simultaneous endometrial adenocarcinoma, and ovarian cancer has been reported to occur in $2-8.5 \%$ of patients with endometrial cancer. Almost $90 \%$ of synchronous tumors identified in the ovary and endometrium were of the endometrioid cell type. There is also a high incidence of ovarian EM cases among synchronous adenocarcinomas originating in the ovary and endometrium $(28,29)$. Based on a series of morphologic and molecular genetic studies, Kurman et al (30) proposed a dualistic model that categorizes various types of ovarian cancer into two groups, type I and type II. The cellular origins of ovarian cancer and the mechanisms by which cancer develops have been long debated. The traditional view of ovarian carcinogenesis is that various tumors are all derived from the ovarian surface epithelium (mesothelium) and that subsequent metaplastic changes lead to development of different cell types (e.g., serous, endometrioid, clear cell, mucinous and transitional cell). However, compelling recent evidence suggests that the vast majority of what appear to be primary ovarian cancers, including serous, endometrioid and clear cell carcinomas, are derived from the fallopian tubes and endometrium and not directly from the ovary. Endometrioid and clear cell carcinomas may develop from malignant transformation of ovarian endometriotic cysts, with secondary involvement from the ovary (31). Studies have reported that malignant transformation of ovarian endometriosis is a continual process. Similar to the eutopic endometrium, ectopic endometrial cells can also transform into atypical hyperplasia and cancer (32). In addition, other authors have demonstrated a higher prevalence 
of endometrial pathology (e.g., endometrial adenocarcinoma, hyperplasia and polyps) when ovarian tumors were considered to originate from endometriosis (33). This indicates a shared molecular pathogenesis in the process of malignant transformation of the eutopic and ectopic endometrium. Although the precise mechanism leading to endometriosis is not clear, it is generally accepted that the eutopic endometrium in women with endometriosis has intrinsic molecular abnormalities (1). Moreover, Rosenblatt et al observed that the strongest apparent protective effects of tubal ligation occurred for clear cell and endometrioid tumors, but there were no differences in the risk for borderline and malignant tumors (34). In a previous study, we detected a higher methylation frequency of hMLH1 in the eutopic endometrium from the EAOC group compared to the EM and CE groups, suggesting that abnormal hMLH1 methylation in the eutopic endometrium from EAOC and malignant transformation of the ectopic endometrium are interrelated (35). Consequently, intrinsic molecular abnormalities in the eutopic endometrium may be the source of malignant transformation of the ectopic endometrium. In the present study, we evaluated the RUNX3 methylation status and protein expression in the eutopic endometrium from three groups. The frequency of epigenetic RUNX3 inactivation in the eutopic endometrium from the EAOC group was higher than in the EM and CE groups. These results verify the hypothesis that intrinsic molecular abnormalities in the eutopic endometrium may be the source of malignant transformation of the ectopic endometrium and that the eutopic and ectopic endometrium may be synchronously affected by RUNX3 hypermethylation. Epigenomic RUNX3 alterations in the eutopic endometrium promoted malignant transformation of the ectopic endometrium. Based on the above conclusion, RUNX3 methylation status and protein expression in the eutopic endometrium could provide early diagnosis of malignant transformation of ovarian EM. Furthermore, the eutopic endometrium is readily available and gene alteration in the eutopic endometrium can be easily detected, which solves difficulties of basic experimentation due to a shortage of malignantly transformed ovarian EM samples.

EM is an estrogen-dependent disease and hyperestrogenism has been associated with malignant transformation of endometriotic cysts. The microenvironment provided by endometriosis facilitates accumulation of excess estrogen. There have been several recent reports that estrogen replacement therapy could lead to malignant transformation of endometrial ectopic foci. Recent studies imply that estrogen imprinting can be mediated by epigenetic mechanisms via ER $\alpha$. Suzuki et al (36) found that the frequency of RUNX3 promoter methylation was higher in ER-positive samples than ER-negative samples. Subramaniam et al (14) also reported that RUNX3 promoter hypermethylation was significantly correlated with positive ER expression in invasive carcinomas. Our data showed that $91.67 \%(11 / 12)$ of ER $\alpha$-positive samples had RUNX3 promoter hypermethylation, while only $38.9 \%(7 / 18)$ of ER $\alpha$-negative samples had detectable hypermethylation of the RUNX3 promoter in malignant ovarian endometriotic cysts from the EAOC group. There was a positive correlation between RUNX3 methylation and ER $\alpha$ protein expression. We postulate that activation of ER signaling by high local concentrations of estrogen and inactivation of RUNX3 protein expression due to promoter methylation are involved in the process of malignant transformation of endometriotic cysts. However, it remains to be determined how estrogen receptor signaling initiates methylation of the RUNX3 promoter. Correlation between RUNX3 methylation and estrogen is a main future research direction in malignant transformation of ovarian EM.

In conclusion, we provide evidence that RUNX3 inactivation by promoter hypermethylation constitutes an early event in the malignant transformation of ovarian EM and is closely related to estrogen metabolism. Our results also suggest that epigenetic inactivation of RUNX3 plays a vital role in the occurrence and development of malignant transformation of ovarian EM. Corresponding changes to the eutopic endometrium indicate that detection of RUNX3 protein expression and methylation status in the eutopic endometrium may be valuable tools for identifying patients with ovarian EM who are at an elevated risk of malignant transformation and could lead to preventative strategies and novel treatment approaches for women who have been diagnosed with EAOC.

\section{Acknowledgements}

The present study was supported by grants from the National Natural Science Foundation of China (nos. 81070467 and 81270675) and the Plan of Free Researchers in Shengjing Hospital.

\section{References}

1. Bulun SE: Endometriosis. N Engl J Med 360: 268-279, 2009.

2. Sampson JA: Endometrial carcinoma of the ovary arising in endometrial tissue in that organ. Arch Surg 10: 1-72, 1925.

3. Kobayashi H, Sumimoto K, Moniwa N, et al: Risk of developing ovarian cancer among women with ovarian endometrioma: a cohort study in Shizuoka, Japan. Int J Gynecol Cancer 17: 37-43, 2007.

4. Stern RC, Dash R, Bentley RC, Snyder MJ, Haney AF and Robboy SJ: Malignancy in endometriosis: frequency and comparison of ovarian and extraovarian types. Int J Gynecol Pathol 20: 133-139, 2001.

5. Yoshikawa H, Jimbo H, Okada S, et al: Prevalence of endometriosis in ovarian cancer. Gynecol Obstet Invest 50 (Suppl 1): $11-17,2000$.

6. Shih Ie M and Kurman RJ: Ovarian tumorigenesis: a proposed model based on morphological and molecular genetic analysis. Am J Pathol 164: 1511-1518, 2004.

7. Park SY, Kwon HJ, Lee HE, et al: Promoter CpG island hypermethylation during breast cancer progression. Virchows Arch 458: 73-84, 2011.

8. Martini M, Ciccarone M, Garganese G, et al: Possible involvement of $h M L H 1, p 16($ INK4a) and PTEN in the malignant transformation of endometriosis. Int J Cancer 102: 398-406, 2002.

9. Levanon D, Negreanu V, Bernstein Y, Bar-Am I, Avivi L and Groner Y: AML1, AML2, and AML3, the human members of the runt domain gene-family: cDNA structure, expression, and chromosomal localization. Genomics 23: 425-432, 1994.

10. Uno S, Zembutsu H, Hirasawa A, et al: A genome-wide association study identifies genetic variants in the CDKN2BAS locus associated with endometriosis in Japanese. Nat Genet 42: 707-710, 2010.

11. Habtemariam B, Anisimov VM and MacKerell AD Jr: Cooperative binding of DNA and CBF $\beta$ to the Runt domain of the $\mathrm{CBF} \alpha$ studied via MD simulations. Nucleic Acids Res 33: 4212-4222, 2005.

12. Li QL, Ito K, Sakakura C, et al: Causal relationship between the loss of RUNX3 expression and gastric cancer. Cell 109: 113-124, 2002 . 
13. Zaidi SK, Sullivan AJ, van Wijnen AJ, Stein JL, Stein GS and Lian JB: Integration of Runx and Smad regulatory signals at transcriptionally active subnuclear sites. Proc Natl Acad Sci USA 99: 8048-8053, 2002.

14. Subramaniam MM, Chan JY, Soong R, et al: RUNX3 inactivation by frequent promoter hypermethylation and protein mislocalization constitute an early event in breast cancer progression. Breast Cancer Res Treat 113: 113-121, 2009.

15. Yoshizaki T, Enomoto T, Fujita M, et al: Frequent inactivation of RUNX3 in endometrial carcinoma. Gynecol Oncol 110: 439-444, 2008.

16. Dhillon VS, Shahid $M$ and Husain SA: $C p G$ methylation of the FHIT, FANCF, cyclin-D2, BRCA2 and RUNX3 genes in Granulosa cell tumors (GCTS) of ovarian origin. Mol Cancer 3: $33,2004$.

17. Kato N, Tamura G, Fukase M, Shibuya H and Motoyama T: Hypermethylation of the RUNX3 gene promoter in testicular yolk sac tumor of infants. Am J Pathol 163: 387-391, 2003.

18. Cheng AS, Culhane AC, Chan MW, et al: Epithelial progeny of estrogen-exposed breast progenitor cells display a cancer-like methylome. Cancer Res 68: 1786-1796, 2008.

19. Oxholm D, Knudsen UB, Kryger-Baggesen N and Ravn P: Postmenopausal endometriosis. Acta Obstet Gynecol Scand 86: 1158-1164, 2007.

20. Scott RB: Malignant changes in endometriosis. Obstet Gynecol 2: 283-289, 1953.

21. Jones PA: The DNA methylation paradox. Trends Genet 15: 34-37, 1999.

22. Kulis $M$ and Esteller M: DNA methylation and cancer. Adv Genet 70: 27-56, 2010.

23. Senthong A, Kitkumthorn N, Rattanatanyong P, Khemapech N Triratanachart $S$ and Mutirangura A: Differences in LINE-1 methylation between endometriotic ovarian cyst and endometriosis-associated ovarian cancer. Int J Gynecol Cancer 24: 36-42, 2014

24. Zochbauer-Muller S, Fong KM, Maitra A, et al: 5' CpG island methylation of the FHIT gene is correlated with loss of gene expression in lung and breast cancer. Cancer Res 61: 3581-3585, 2001.

25. Baylin SB and Herman JG: DNA hypermethylation in tumorigenesis: epigenetics joins genetics. Trends Genet 16: 168-174, 2000 .
26. Jones PA and Baylin SB: The fundamental role of epigenetic events in cancer. Nat Rev Genet 3: 415-428, 2002.

27. Wang H, Feng Y, Bao Z, et al: Epigenetic silencing of KAZALD1 confers a better prognosis and is associated with malignant transformation/progression in glioma. Oncol Rep 30: 2089-2096, 2013.

28. Falkenberry SS, Steinhoff MM, Gordinier M, Rappoport S, Gajewski W and Granai CO: Synchronous endometrioid tumors of the ovary and endometrium. A clinicopathologic study of 22 cases. J Reprod Med 41: 713-718, 1996.

29. Zaino R, Whitney C, Brady MF, DeGeest K, Burger RA and Buller RE: Simultaneously detected endometrial and ovarian carcinomas - a prospective clinicopathologic study of 74 cases: a Gynecologic Oncology Group study. Gynecol Oncol 83: 355-362, 2001.

30. Kurman RJ and Shih IeM: Pathogenesis of ovarian cancer: lessons from morphology and molecular biology and their clinical implications. Int J Gynecol Pathol 27: 151-160, 2008.

31. Martin DC: Cancer and endometriosis: do we need to be concerned? Semin Reprod Endocrinol 15: 319-324, 1997.

32. Vigano P, Somigliana E, Chiodo I, Abbiati A and Vercellini P: Molecular mechanisms and biological plausibility underlying the malignant transformation of endometriosis: a critical analysis. Hum Reprod Update 12: 77-89, 2006.

33. Van Gorp T, Amant F, Neven P, Vergote I and Moerman P: Endometriosis and the development of malignant tumours of the pelvis. A review of literature. Best Pract Res Clin Obstet Gynaecol 18: 349-371, 2004.

34. Rosenblatt KA and Thomas DB: Reduced risk of ovarian cancer in women with a tubal ligation or hysterectomy. The World Health Organization Collaborative Study of Neoplasia and Steroid Contraceptives. Cancer Epidemiol Biomarkers Prev 5: 933-935, 1996.

35. Ren F, Wang D, Jiang Y and Ren F: Epigenetic inactivation of hMLH1 in the malignant transformation of ovarian endometriosis. Arch Gynecol Obstet 285: 215-221, 2012.

36. Suzuki M, Shigematsu H, Shames DS, et al: DNA methylationassociated inactivation of TGF $\beta$-related genes DRM/Gremlin, RUNX3, and HPPI in human cancers. Br J Cancer 93: 1029-1037, 2005. 